\title{
Editorial
}

\section{Clinical Decisions in Acute Patients: ACS-POCT-Hypertension and Biomarkers}

\author{
Martin Möckel, ${ }^{1}$ Christian Hamm, ${ }^{2}$ Evangelos Giannitsis, ${ }^{3}$ \\ Abdelouahab Bellou, ${ }^{4}$ and Julia Searle ${ }^{1}$ \\ ${ }^{1}$ Division of Emergency Medicine/CPU, Charité-University Medicine Berlin, Campus Virchow-Klinikum and Campus Charité Mitte, \\ 13353 Berlin, Germany \\ ${ }^{2}$ Kerckhoff Heart and Thorax Center, Bad Nauheim and Medical Clinic I, University of Giessen, 61231 Bad Nauheim, Germany \\ ${ }^{3}$ Department of Internal Medicine III, Cardiology, University Hospital Heidelberg, 69120 Heidelberg, Germany \\ ${ }^{4}$ European Society for Emergency Medicine, EuSEM and Emergency Medicine Department, Faculty of Medicine, \\ Rennes University Hospital, 35033 Rennes, France \\ Correspondence should be addressed to Martin Möckel; martin.moeckel@charite.de
}

Received 16 June 2013; Accepted 16 June 2013

Copyright (C 2013 Martin Möckel et al. This is an open access article distributed under the Creative Commons Attribution License, which permits unrestricted use, distribution, and reproduction in any medium, provided the original work is properly cited.

This edition presents a number of papers derived from the $3 \mathrm{rd}$ Symposium "Acute Cardiology" of the Division of Emergency Medicine at the Charité Berlin, which was held in Berlin, Germany, in October 2012. The symposium highlighted new developments in the field of biomarker research in acute cardiology. This included biomarker strategies for process optimization in acute coronary syndrome, acute heart failure, arterial hypertension, and biomarker monitoring in antithrombotic therapy and renal denervation therapy.

Acute and emergency medicine is developing into an independent clinical and scientific speciality. This is especially important, as increasing subspecialization in other medical specialist areas causes gaps in the care of acutely ill patients.

Cardiovascular emergency care is one of the areas where new pathways for process orientation and innovative strategies are most important to improve patient management. This will most likely include new pathophysiology-oriented biomarker strategies.

The use and interpretation of hs troponins in clinical practice is one of the big challenges in acute cardiology these days. The paper by K. Thygesen and J. Searle summarizes the recently updated definition of acute myocardial infarction in a comprehensive way. The paper by $\mathrm{H}$. A. Katus and E. Giannitsis gives clear instructions on how to use hs troponin in clinical practise. Hs troponin has evolved as a "normal" marker with $100 \%$ cardiospecificity, improved early sensitivity, but lack of specificity for coronary origin of elevated levels in acute heart disease. As a consequence, not every troponin elevation, even if a type $2 \mathrm{MI}$ is diagnosed, requires immediate coronary angiography. Nevertheless, every troponin elevation should trigger a thorough diagnostic workup [1]. In the light of potential renal complications as highlighted by the contribution of P. B. Persson, the evaluation of small troponin elevations has to be handled with care and perhaps some patients profit more from early imaging than cardiac catheterization. The use of biomarkers, therefore, requires as a basis a thorough clinical examination and an expertise judgement by physicians trained in acute cardiology. In addition, to provide high-quality treatment, diagnostic and subsequent invasive testing needs a clear process orientation with a high level of standardization as outlined in recent publications $[2,3]$.

In the acute setting, procedural aspects play a major role which is described in papers by V. Lindenau-Stockfisch et al. and M. Hansen-Nord. It becomes clear that emergency cardiovascular care needs to be process and patient oriented, to achieve optimal outcomes. Whether this is facilitated by new markers like copeptin, mr-proADM, and mr-proANP and by point of care devices remains a matter of debate. Specifically, it seems to be an open question whether POCT 


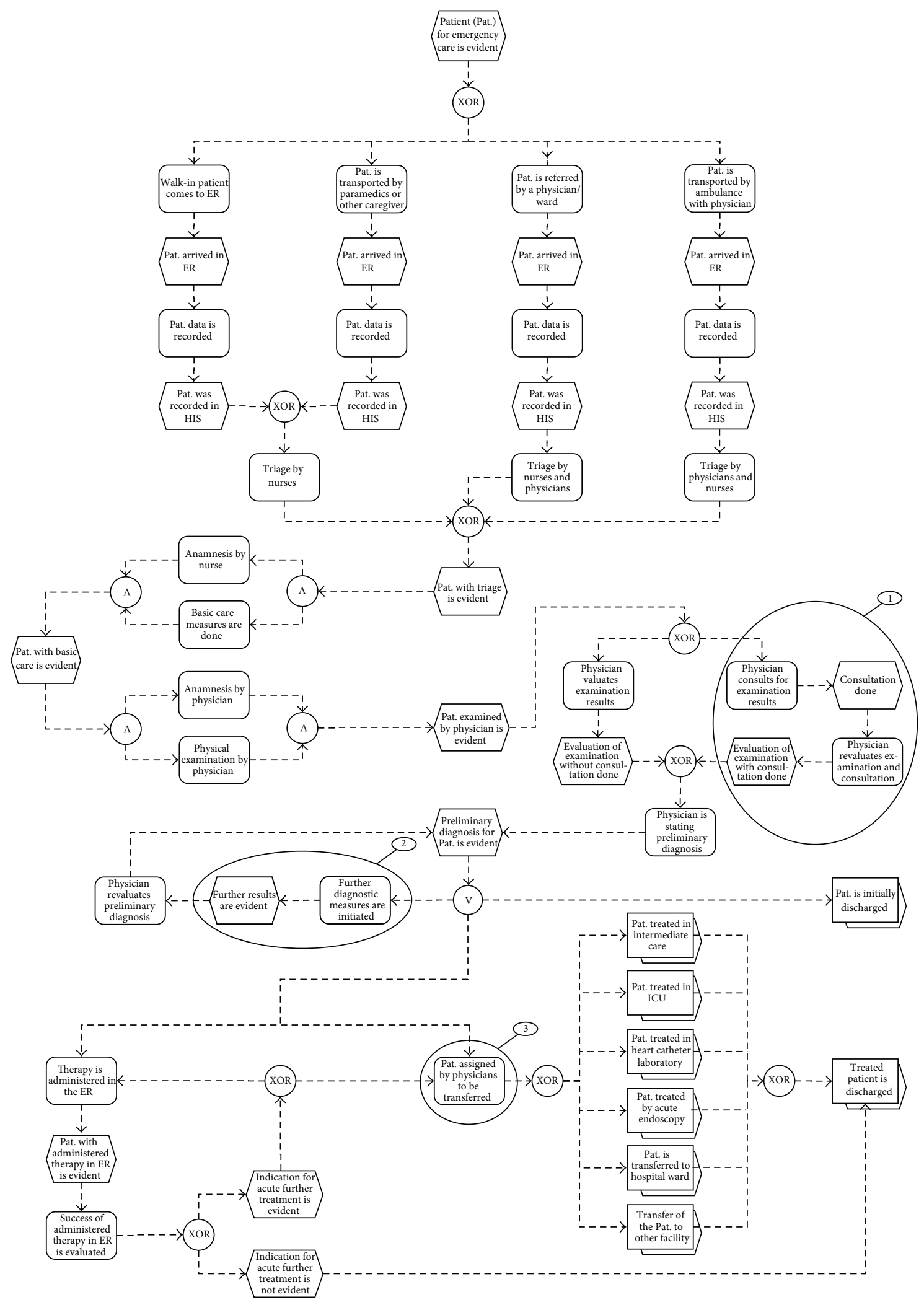

FIGURE 1: Event-process-chain of acute emergency care. Details of the notation are published elsewhere (Vollert et al. [2]). From M. Möckel and J. O. Vollert. Processes in acute and emergency medicine, unpublished data. 
has benefits over central-laboratory-based troponin measurements, which may have a much longer turn-around time and thus counteract in part the potential benefit of higher early sensitivity of hs troponin. Additionally, the concept of delta changes for serial troponin measurements is challenged by data presented by O. Hammarsten. At the moment, POCT can be safely used when an appropriate assay is utilized and the observation period is at least 6 hours. New data which will be available in the very near future (BIC-8 study on early ruleout with copeptin and troponin; NCT01498731) will provide new insights into the optimal early decision strategies.

As a summary, the processes of acute care are described in Figure 1 (event process chain). Critical processes are circled (circles 1-3). In particular the step "physician (specialist) consultation" (circle 1) requires a lot of time and opens potential for new biomarker testing.

Martin Möckel Christian Hamm Evangelos Giannitsis Abdelouahab Bellou Julia Searle

\section{References}

[1] M. Vafaie, M. Biener, M. Mueller et al., "Analytically false or true positive elevations of high sensitivity cardiac troponin: a systematic approach," Heart, 2013.

[2] J. Vollert, C. Hamm, A. G. SOP Kardiologie, and M. Möckel, "Prozessorientierte und standardisierte Umsetzung von DGKLeitlinien: Vorgehen beim ST-Strecken-Hebungsinfarkt," Der Kardiologe, vol. 3, pp. 388-405, 2009.

[3] M. Möckel, J. Vollert, C. Hamm, and A. G. SOP Kardiologie, "'Standard operating procedures' für den akuten ST-Streckenhebungsinfarkt: Anwendung prozessbasierter Grundsätze," Der Kardiologe, vol. 4, pp. 124-134, 2010. 

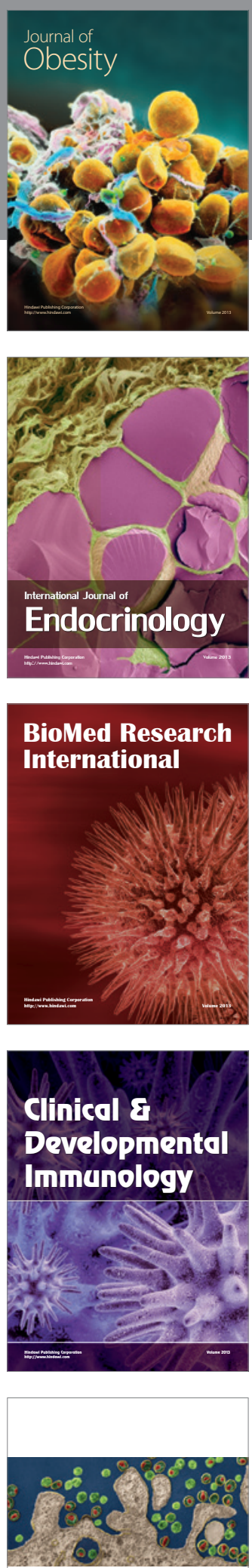

ISRN

AIDS
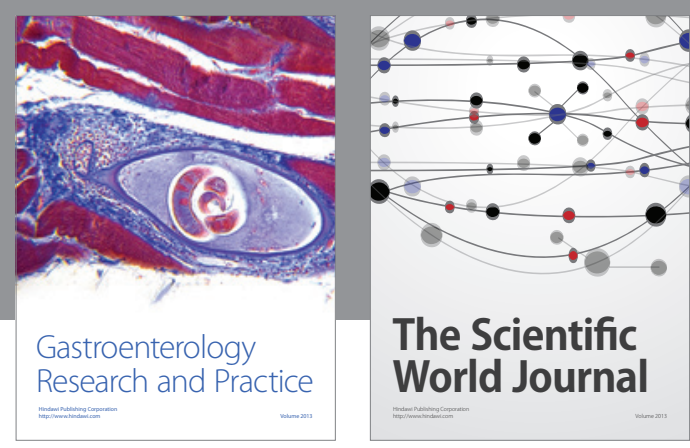

The Scientific World Journal
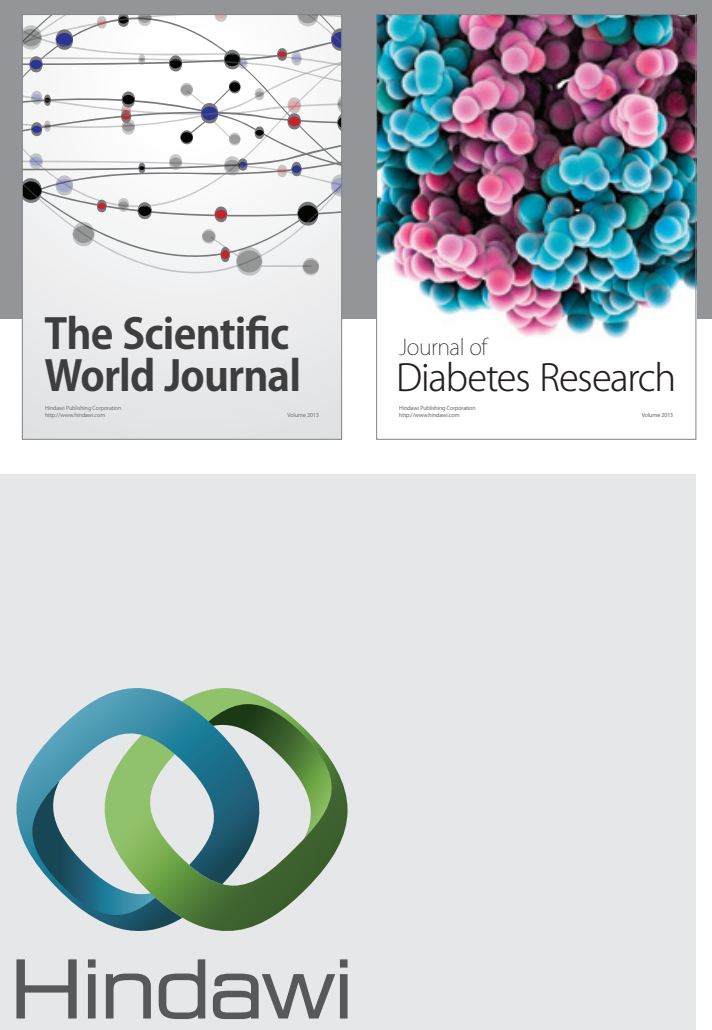

Submit your manuscripts at

http://www.hindawi.com
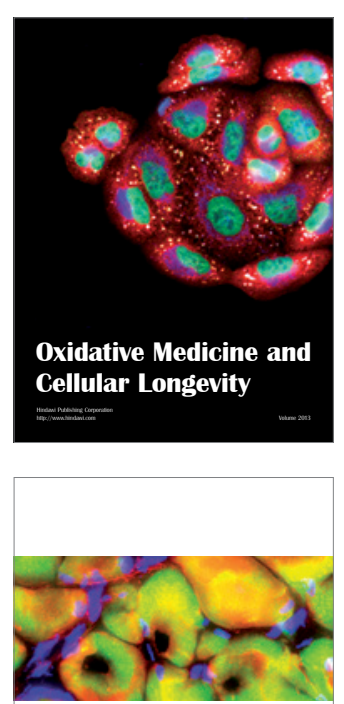

ISRN

Biomarkers
MEDIATORS

INFLAMMATION
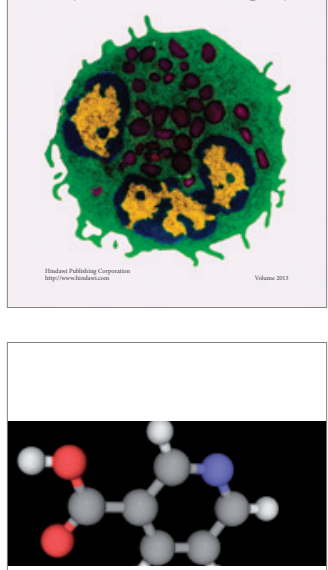

ISRN

Addiction
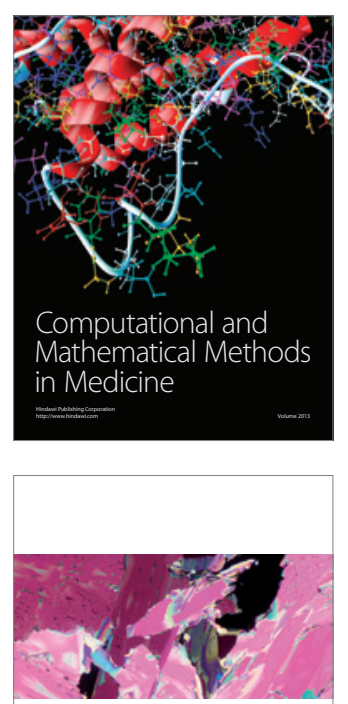

ISRN

Anesthesiology
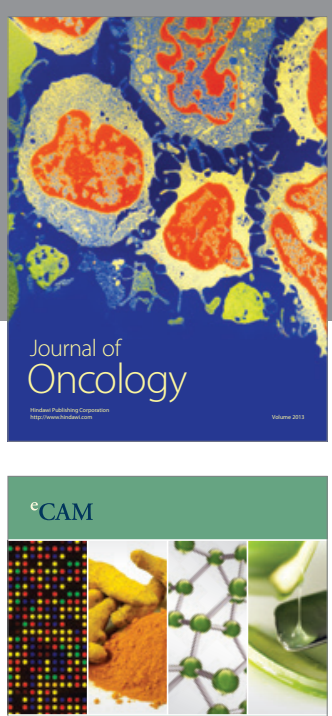

Evidence-Based

Complementary and

Alternative Medicine
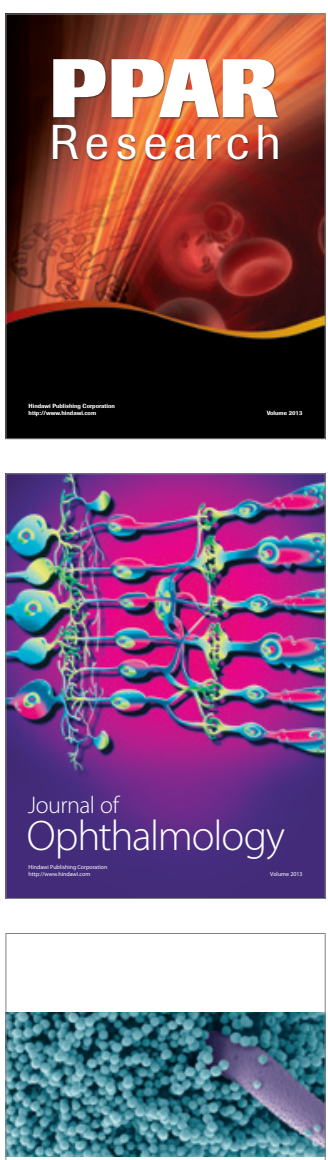

ISRN

Allergy 


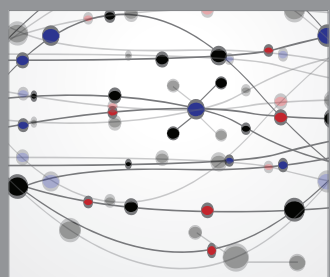

The Scientific World Journal
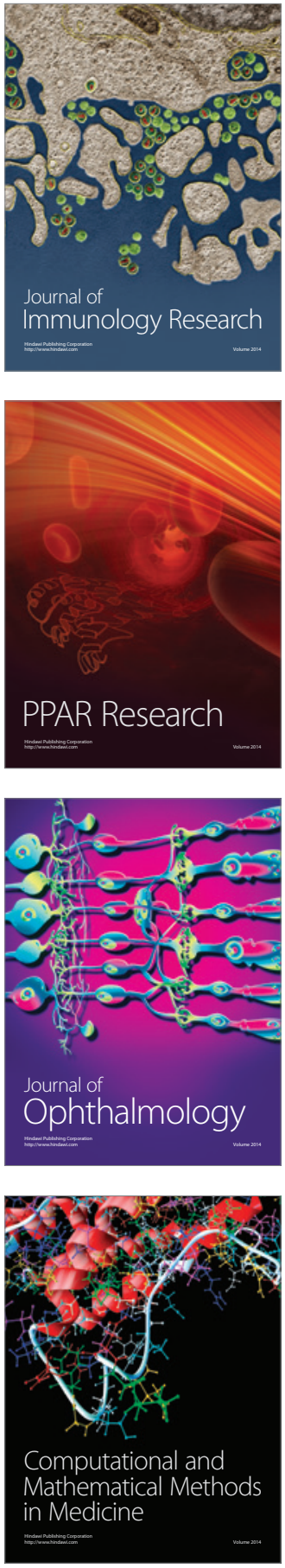

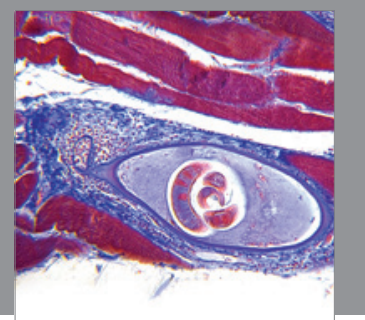

Gastroenterology

Research and Practice
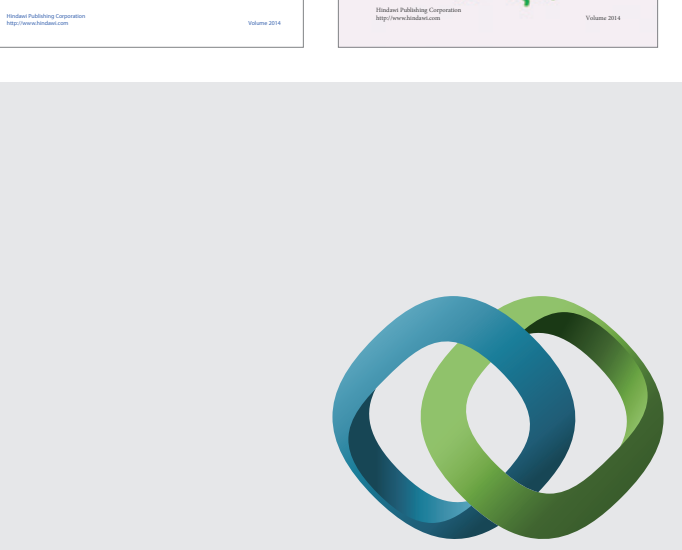

\section{Hindawi}

Submit your manuscripts at

http://www.hindawi.com
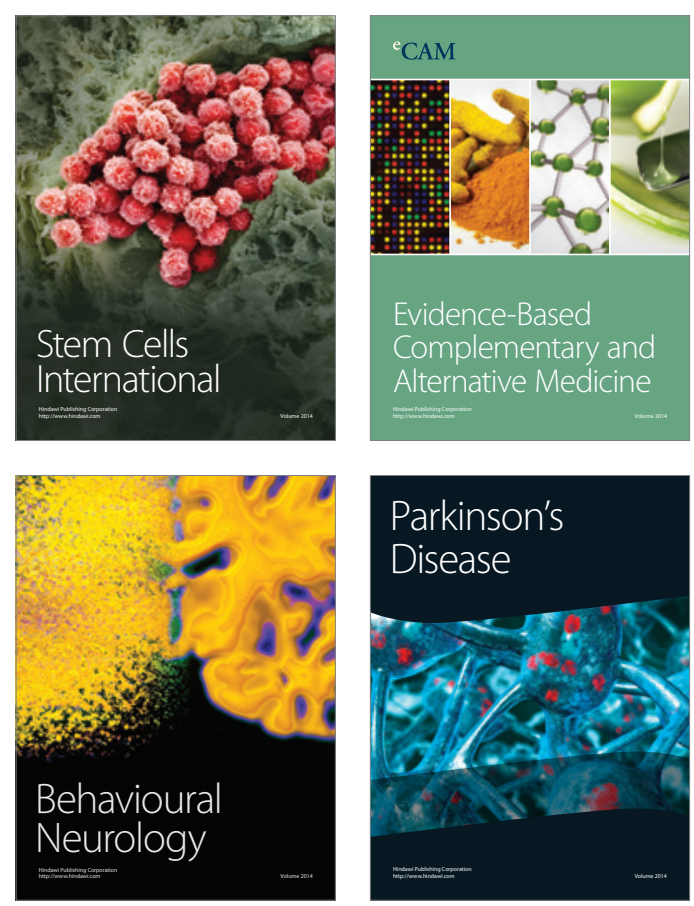

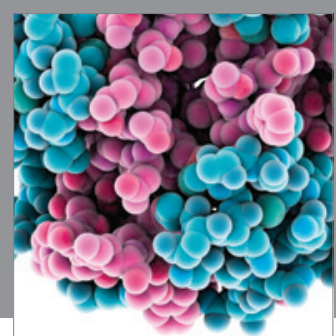

Journal of
Diabetes Research

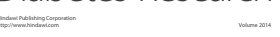

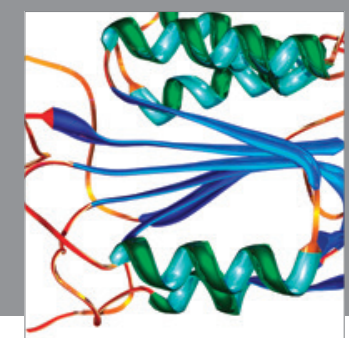

Disease Markers
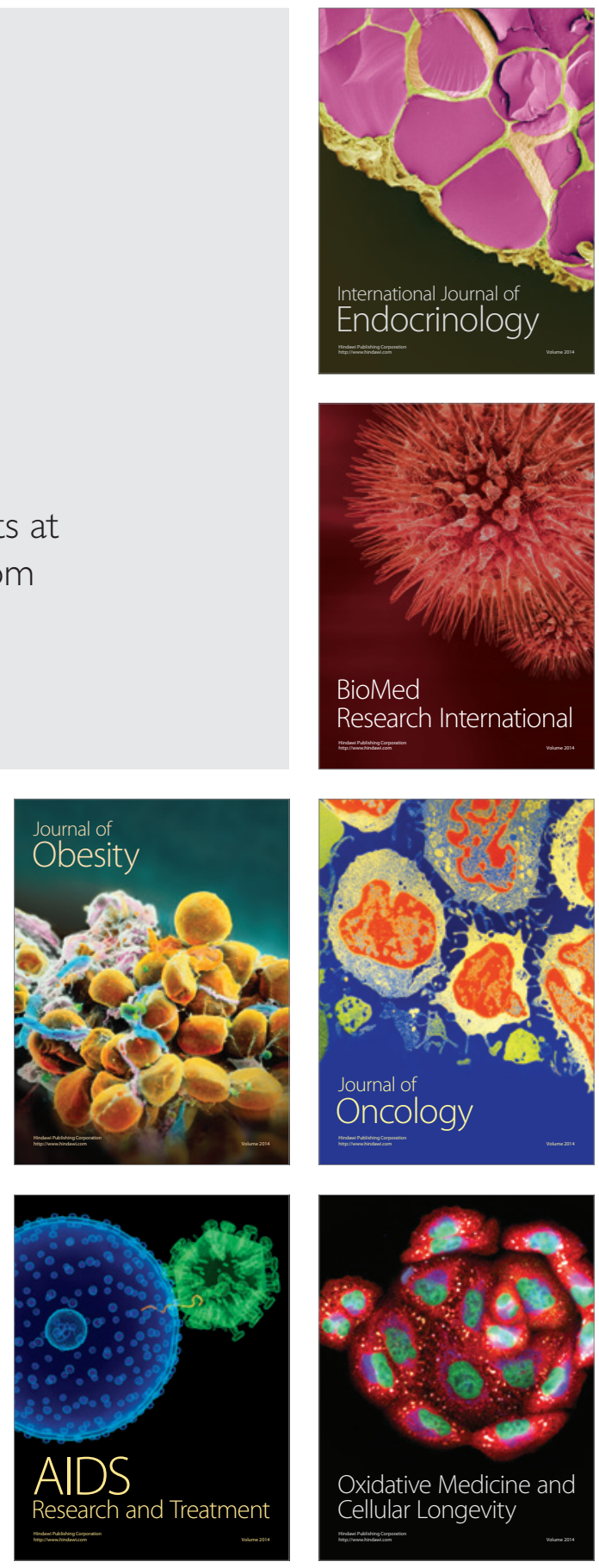